Ann. Biol, anim. Bioch. Biophys., I975, 15 (3), 517-523.

\title{
SURVIE DES SPERMATOZOÏDES DE VERRAT APRES DÉCONGÉLATION. EFFET DU RYTHME DE COLLECTES, DE LA CONCENTRATION ET DU TAUX DE GLYCÉROL
}

\author{
M. PAQUIGNON et M. COUROT* \\ avec Ia collaboration technique de J. GaUtier \\ I. T. P., 149, rue de Bercy, \\ 75579 Paris Cedex 12 \\ * Station de Physiologie de la Reproduction, \\ Centre de Recherches de Tours, I. N.R.A., \\ Nouzilly, 37380 Monnaie
}

\begin{abstract}
RÉSUMÉ
Ce travail a porté sur la congélation du sperme de verrat récolté I ou 2 fois par semaine. L'influence du taux de dilution de la semence et de la quantité de glycérol dans le dilueur a été étudiée in vitvo, en estimant le pourcentage de spermatozoïdes mobiles après décongélation et incubation du sperme 3 heures à $37^{\circ} \mathrm{C}$. Des récoltes bihebdomadaires permettent d'augmenter de I3,6 p. Ioo la quantité de spermatozoïdes récoltés et de 25,2 p. roo la quantité de spermatozoïdes mobiles obtenus après décongélation. Il y a une interaction positive entre le taux de dilution et le taux de glycérol par spermatozoïde sur la reviviscence des spermatozoïdes. L'effet de la dilution est supérieur à celui de glycérol. Une concentration de $400 \cdot 10^{6} \mathrm{spz} / \mathrm{ml}$ et un taux final de glycérol de 2 p. Ioo donnent la meilleure survie après 3 heures d'incubation. La réanimation et la survie sont plus élevées avec du sperme récolté en hiver.
\end{abstract}

\section{INTRODUCTION}

La composition du dilueur de congélation, la vitesse de refroidissement et la température de glycérolisation de la semence de verrat ont une influence significative sur la survie des spermatozoïdes après décongélation. Mais dans les meilleures conditions, le taux de reviviscence obtenu avec une concentration élevée en spermatozoïdes 
(I $3000 \cdot \mathrm{IO}^{6} \mathrm{spz} / \mathrm{ml}$ ) est encore faible (PAQUiGNon et al., I974). Sachant que 1'augmentation de la concentration en spermatozoïdes est néfaste (RoHLOFF, I972; GraHAM, I972), plusieurs auteurs ont étudié l'influence du taux de dilution sur le pourcentage de réanimation soit en fonction d'un taux initial de glycérol constant (ROHLOFF, I972) soit en fonction d'un taux final constant (SALAMON, I973).

Aucun travail n'a porté sur l'effet de la dilution avec une concentration constante de glycérol par spermatozoïde ; on ne connaît pas l'effet propre de la dilution ni celui du glycérol. Or, avec un dilueur non glycérolé, l'augmentation de la dilution améliore le taux de spermatozoïdes réanimés (GRAHAM, I972), et pour une même dilution l'augmentation du taux de glycérol améliore la survie des spermatozoïdes après décongélation (WILMUT et al., I972).

L'objet du travail était de définir les conditions optimales de dilution du sperme en déterminant le rôle respectif du taux de dilution et de la concentration du glycérol par spermatozoïde dans 1'amélioration du pourcentage de spermatozoïdes réanimés dès la décongélation et après incubation.

\title{
MATÉRIEL ET MÉTHODES
}

\begin{abstract}
Quatre verrats ont été utilisés dans deux expériences l'une entre janvier et mars avec alternativement $\mathrm{I}$ ou 2 récoltes de semence par semaine et l'autre en juin et juillet avec 2 récoltes par semaine. Douze éjaculats ont servi pour chaque expérience ( 4 verrats $\times 3$ éjaculats ; un seul éjaculat était congelé dans le cas des récoltes bihebdomadaires). L'éjaculat entier était filtré sur gaze et centrifugé à $800 \mathrm{~g}$ pendant I $5 \mathrm{mn}$ à $30^{\circ} \mathrm{C}$. Le surnageant était rejeté et les spermatozoïdes du culot dilués une première fois avec un dilueur jaune d'œuf glucose (Polge et al., 1970) à $30^{\circ} \mathrm{C}$ de telle sorte que la concentration en spermatozoïdes était de $2400 \cdot 10^{6} \mathrm{spz} / \mathrm{ml}$. La température était ensuite abaissée de $30^{\circ} \mathrm{C}$ à $15^{\circ} \mathrm{C}$ en I heure et maintenue à $15^{\circ} \mathrm{C}$ pendant 4 heures. La semence était alors diluée une deuxième fois avec le même dilueur additionné de glycérol. Le taux de dilution était ajusté pour que les concentrations en spermatozoïdes soient de I 600,800,400, 200 $10^{6}$ $\mathrm{spz} / \mathrm{ml}$ dans la première expérience et $800,400,200 \cdot{ }^{6} 0^{6} \mathrm{spz} / \mathrm{ml}$ dans la deuxième. Les taux de glycérol étaient exprimés en picomole/spz: $0,085,0,17,0,34,0,68$ picomole/spz dans la première expérience et en volume relatif final i p. Ioo, 2 p. I OO, 4 p. I oo, 8 p. I oo dans la deuxième. La température du mélange était abaissée de $15^{\circ} \mathrm{C}$ à $5^{\circ} \mathrm{C}$ en I heure. La semence était alors congelée en pastilles de $\mathrm{o}, \mathrm{I} \mathrm{ml}$ sur de la glace carbonique puis transferrée dans l'azote liquide. La décongélation était réalisée à $5^{\circ} \mathrm{C}$ dans le dilueur I. N. R. A.-I. T. P. (I/5 en volume) (PAQUignon et al., i973). La qualité de la semence était appréciée au microscope à contraste de phase, par le pourcentage de spermatozoïdes vivants après décongélation et 3 heures d'incubation à $37^{\circ} \mathrm{C}$. L'examen des différents échantillons était pratiqué sans en connaître l'origine. I.'analyse de variance a été réalisée après transformation des pourcentages en Arc $\sin \sqrt{\mathrm{P} / \mathrm{IoO}}$.
\end{abstract}

\section{RÉSULTATS}

\section{I. - Infuence du rythme de collecte}

Les caractéristiques de la semence collectée une et deux fois par semaine sont présentées dans le tableau I. Le volume de l'éjaculat, sa concentration en spermatozoïdes et le nombre total de spermatozoïdes par éjaculat sont significativement réduits $(\mathrm{P} \leqslant \mathrm{O}, \mathrm{OI})$ dans le cas des récoltes bihebdomadaires. Cependant, la quantité 
de spermatozoïdes récoltés chaque semaine est accrue de $13,6 \mathrm{p}$. Ioo $\left(94 \cdot{ }^{\prime} \mathrm{I}^{9}\right.$ contre $\left.82 \cdot \mathrm{IO}^{9}\right)$ et le taux de spermatozoïdes réanimés dès la décongélation ou après 3 heures d'incubation est significativement plus élevé avec detux récoltes par semaine $(P \leqslant 0,0$ I $)$

TABI,EAU I

Caractéristiques des éjaculats de verrats collectés une ou deux fois par semaine

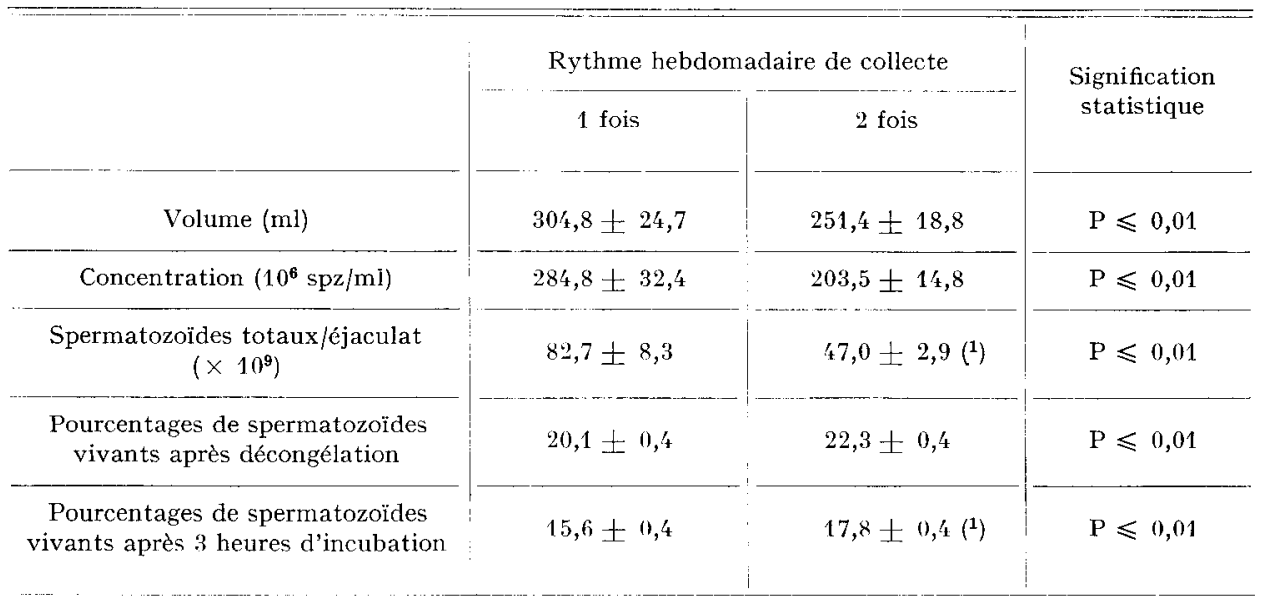

(1) Rapportés à la quantité de spermatozoïdes disponibles par semaine, ces chiffres traduisent une augmentation de 13,6 p. 100 et de 25,9 p. 100 du nombre de spermatozoïdes totaux et de spermatozoïdes mobiles après dégel et incubation lorsque l'on passe de 1 à 2 récoltes hebdomadaires.

\section{2. - Influence de la concentration en spermatozoïdes et du taux de glycérol}

L'analyse de variance révèle une interaction positive $(P \leqslant 0,0 I)$ entre le taux de dilution et le taux de glycérol par spermatozoïde après décongélation et 3 heures d'incubation. L'effet de la diminution de la concentration en spermatozoïdes est supérieur à celui de l'augmentation du taux de glycérol.

\section{Après décongélation.}

Une concentration de $\mathrm{spz} / \mathrm{m} 1$ décroissante provoque une augmentation significative du taux de reviviscence que le glycérol soit ajusté par rapport aux spermatozoïdes $(\mathrm{P} \leqslant \mathrm{O}, \mathrm{OI})\left(\mathrm{I} 3,6 \mathrm{p}\right.$. IOO et $25,5 \mathrm{p}$. IOO respectivement pour I 600 et $200 \cdot \mathrm{IO}^{6}$ $\mathrm{spz} / \mathrm{ml}$ ) (fig. I) ou au volume de dilueur $(\mathrm{P} \leqslant 0,05)\left(\mathrm{I}_{7}, 2 \mathrm{p}\right.$. Ioo et $2 \mathrm{I}, \mathrm{I}$ p. Ioo respectivement pour 800 et $200 \cdot \mathrm{IO}^{6} \mathrm{spz} / \mathrm{ml}$ (fig. 2). Une concentration de glycérol inférieure à $0, \mathrm{I} 7$ picomole/spz provoque un abaissement significatif $(\mathrm{P} \leqslant 0,05)$ du nombre de spermatozoïdes vivants (fig. I) (I9,2 p. I00 contre $2 \mathrm{I}, 3$ p. I00, 22,7 p. I00, 2I,7 p. IOO respectivement pour $0,085,0, \mathrm{I} 7,0,34,0,68 \mathrm{pmole} / \mathrm{spz}$ ). Toutefois, une concentration élevée en spermatozoïdes ( $\mathrm{I} 600 \cdot \mathrm{IO}^{6} \mathrm{spz} / \mathrm{ml}$ ) associée au plus fort taux de glycérol $(0,68 \mathrm{pmole} / \mathrm{spz}$ ) donne un très faible taux de spermatozoïdes réanimés (fig. I). Un taux final de glycérol supérieur à $4 \mathrm{p}$. Ioo abaisse significativement la reviviscence des spermatozoïdes (fig. 2) $(22,0$ p. IOO, 22,8 p. I0O, 20,3 p. IOO contre II, 8 p. IOO respectivement pour I p. IOo, 2 p. IOO, 4 p. Ioo et 8 p. IOO). 

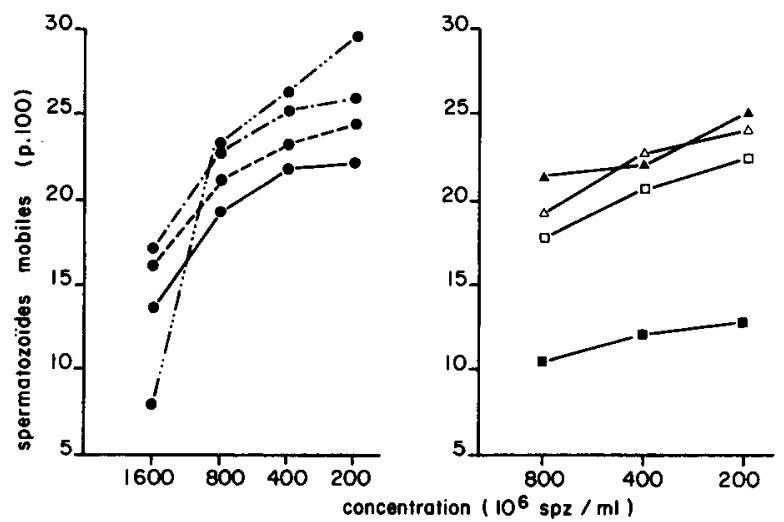

FIG. I

FIG. 2

FIG. I. - Effets du taux de glycérol par spermatozoüde

et de la concentration sur le pourcentage de spermatozoïdes vivants après décongélation

Taux de glycérol

par spermatozoïde

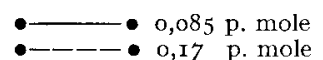

-...- 0,34 p. mole $n=24$

FIG. 2. - Effet du taux de glycérol final et de la concentration en spermatozoïdes sur le pourcentage de spermatozoïdes mobiles après décongélation

Taux final de

glycérol p. I 100

$n=12$
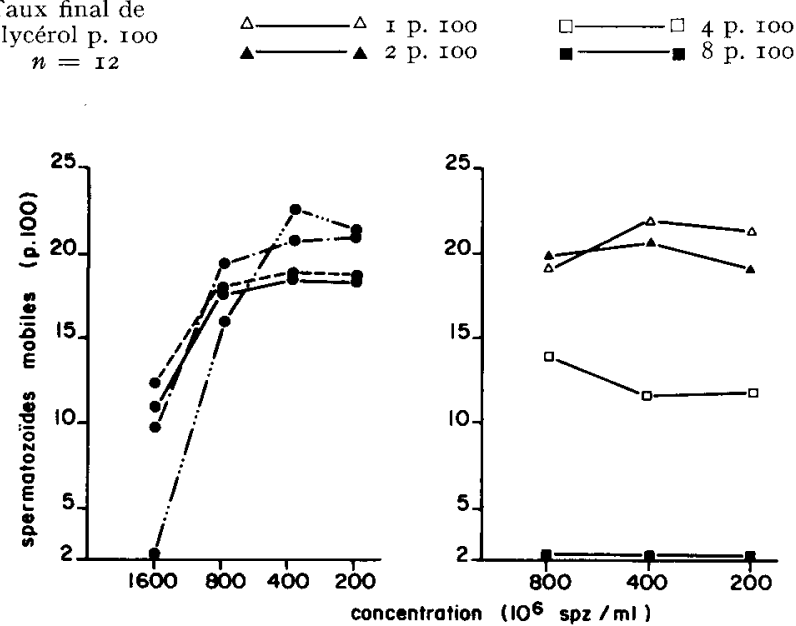

FIG. 3

FIG. 4

Fig. 3. - Effet du taux de glycérol par spermatozoüde

et de la concentration en spermatozoïde sur la survie des spermatozoüdes après 3 heures d'incubation à $37^{\circ} \mathrm{C}$

Taux de glycérol

par spermatozoïde

$$
n=24
$$

$\bullet-0,085$ p. mole

o,ry p. mole $\bullet-\cdots \cdot-0,34$ p. mole

- $\cdots, 68 \mathrm{p}$. mole

FIG. 4. - Effet du taux de glycérol final et de la concentration en spermatozoïdes sur la survie des spermatozö̈des après 3 heures d'incubation à $37{ }^{\circ} \mathrm{C}$

Taux final de glycérol p. Ioo

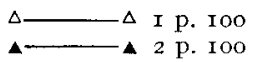

$n=\mathrm{I} 2$

[- 4 p. IOO

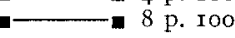


Après incubation.

Pour un même taux de glycérol par cellule, la survie des spermatozoïdes à l'incubation est significativement meilleure $(P \leqslant 0,0 I)$ avec une concentration égale ou inférieure à $400 \cdot 10^{k} \mathrm{spz} / \mathrm{ml}$ (fig. 3). Si le glycérol est ajusté en fonction du volume final pour un taux de I à 8 p. Ioo, la concentration du sperme, dans la limite de 800 à $200 \cdot 10^{6} \mathrm{spz} / \mathrm{ml}$ semble ne pas avoir d'influence sur le maintien de la survie des spermatozoïdes (fig. 4).

Mais le taux de glycérol influe notablement sur la survie des spermatozoïdes à l'incubation : l'effet défavorable de la plus forte concentration en glycérol par spermatozoïde associée à la plus faible dilution du sperme est confirmée (fig. 3). Avec le glycérol ajusté au volume final de dilution, il apparaît qu'un taux supérieur à 2 p. roo diminue significativement $(\mathrm{P} \leqslant 0,0 \mathrm{I})$ le pourcentage de survie des spermatozoïdes à l'incubation $(20,8 \mathrm{p}$. Ioo et $19,8 \mathrm{p}$. Ioo pour I et $2 \mathrm{p}$. Ioo de glycérol contre I2,4 et 2,3 p. roo pour 4 et 8 p. Ioo de glycérol) (fig. 4).

\section{3. - Infuence de la saison sur la qualité des spermatozoïdes après congélation}

La comparaison des résultats obtenus en hiver et en été montre que le taux de spermatozoïdes vivants après décongélation et 3 heures d'incubation est significativement plus élevé en hiver qu'en été $(\mathrm{P} \leqslant \mathrm{O}, \mathrm{OI})(25,7 \mathrm{p}$. Ioo et $2 \mathrm{I}, 6 \mathrm{p}$. Ioo contre $2 \mathrm{I}, 2$ p. Ioo et I $8,6 \mathrm{p}$. Ioo respectivement en hiver et en été). Ceci traduit une meilleure qualité initiale de la semence récoltée en hiver.

\section{DISCUSSION}

La quantité totale de semence récoltée chaque semaine est plus élevée avec deux qu'avec une seule collecte hebdomadaire ( + I3,6 p. Ioo). Ce résultat confirme les travaux de Johnson et al. (I969), du Mesnil, du Buisson et al. (I970), Swierstra (I973). L'augmentation significative du taux de spermatozoïdes vivants après décongélation pour des collectes bihebdomadaires permet d'améliorer la production hebdomadaire de spermatozoïdes mobiles de 25,9 p. Ioo. Cette augmentation résulte probablement de la meilleure qualité de la semence récoltée dans ces conditions.

L'effet néfaste de concentrations élevées ( $\mathrm{I} 600 \cdot \mathrm{IO}^{6} \mathrm{spz} / \mathrm{ml}$ ) sur le taux de spermatozoides réanimés confirme les observations de RoHLOFF (I972) et Graham (I972). A l'inverse, quel que soit le taux de glycérol, une concentration de $200 \cdot \mathbf{I O}^{6}$ $\mathrm{spz} / \mathrm{ml}$ donne après dégel le taux de spermatozoïdes réanimés le plus élevé. Cependant, après 3 heures d'incubation le meilleur taux de survie est obtenu avec une concentration de $400 \cdot 10^{6} \mathrm{spz} / \mathrm{ml}$. De faibles concentrations $\left(\leqslant 96 \cdot 10^{6} \mathrm{spz} / \mathrm{ml}\right)$ augmentent la sensibilité des spermatozoïdes au choc au froid (PURSEL et al., I973). L'amélioration du taux de réanimation pour des taux croissants de glycérol par spermatozoïde confirme les travaux de WILmuT et al. (I973). Cependant, il existe un optimum dans la concentration en glycérol par spermatozoïde. Des concentrations égales ou supérieures à 0,34 pmole par spermatozoïde associées à de fortes concentrations en spermatozoïdes, I 600 et $800 \cdot \mathrm{IO}^{6} \mathrm{spz} / \mathrm{ml}$, provoquent une forte chute de la survie après 3 heures d'incubation. L'effet de l'augmentation du taux de dilution est supérieur à celui du taux de glycérol par spermatozoïde. Ainsi, la concentration par spermatozoïde des autres 
constituants du dilueur (glucose-jaune d'œuf) semble aussi importante que celle du glycérol dans la protection des spermatozoïdes de verrat au cours du processus de congélation. Il vient d'ailleurs d'être démontré que le glucose comme le glycérol sont métabolisés par les spermatozoïdes de verrat pour entrer dans la composition des lipides membranaires (DAcheux et Pagurgnon, I974). L'abaissement du taux de survie pour des taux de glycérol supérieur à $2 \mathrm{p}$. Ioo confirme les travaux de VISSER et SALAmon (I974). Le taux final de 2 p. Ioo est donc une limite supérieure qu'il ne faut pas dépasser sans risque d'augmenter la décharge de la transaminase glutamique-oxalacétique (GOT) de la cellule (BOWER et al., I973), d'abaisser le taux d'acrosomes normaux (GRAHAm et al., I972) et le taux d'œufs fécondés, même après insémination par voie chirurgicale (WILmUT et al., I972).

Ainsi, l'utilisation de très faible taux de glycérol (EInARsson et al., I973; Paquignon et al., I975; PURSEL, et al., I974) est probablement un des éléments du succès actuel de l'I. A. porcine avec du sperme congelé.

\section{CONCLUSION}

Dans la pratique de l'insémination artificielle avec du sperme frais, les verrats sont collectés I fois par semaine. Or, l'emploi des techniques de congélation pose des problèmes de quantité et de qualité des spermatozoïdes en raison des faibles taux de reviviscence obtenus. Ce travail a permis de les résoudre en partie : d'une part, des récoltes bihebdomadaires permettent d'obtenir une semence de meilleure qualité; d'autre part, une dilution ajustant la concentration finale du sperme à une valeur comprise entre 400 et $800 \cdot 10^{6} \mathrm{spz} / \mathrm{ml}$ avec un taux final de glycérol de $2 \mathrm{p}$. Ioo donne les meilleures conditions de conservation de la semence. Il reste à confirmer que ces améliorations observées in vitro donneront le meilleur taux de fécondance : on peut le penser puisque nous avons vérifié à propos des moments de glycérolisation et des vitesses de refroidissement de la semence, qu'une meilleure survie in vitro est associée à une meilleure fécondance (PAQUignon et al., I974; PAQUignon et Courot, I975).

$$
\text { Reçu pour publication en février } 1975 .
$$

\section{REMERCIEMENTS}

Ce travail a été réalisé grâce à un financement du F. O. R. M. A., versé au titre d'une convention passée entre cet organisme, l'I. T. P. et l'I. N. R. A. dans le cadre du programme de rationalisation de la production porcine.

\section{SUMMARY}

BOAR SPERMATOZOA SURVIVAL AFTER THAWING.

EFFECT OF COLLECTION RHYTHM, CONCENTRATION AND AMOUNT OF GLYCEROL,

This report concerns deep-freezing of boar semen following two collection frequencies : one or two ejaculates per week. The influence of dilution rate and quantity of glycerol in the diluent has been studied in vitro by estimating the percentage of motile sperm after thawing and three hours of incubation at $37^{\circ} \mathrm{C}$. With two ejaculates per week, the quantity of spermatozoa collected 
and the number of motile sperm are increased by 13.6 and $25.9 \mathrm{p}$. roo, respectively. There is a positive interaction between the dilution rate and the glycerol level per spermatozoa on the revival rate of spermatozoa. The dilution effect is greater than that of glycerol. The best survival after three hours of incubation is obtained at a concentration of $400 \cdot 10^{6} \mathrm{spz} / \mathrm{ml}$ of diluted semen and a 2 p. Ioo final concentration of glycerol. Both revival and survival rates are higher with sperm collected in winter.

\section{RÉFÉRENCES BIBLIOGRAPHIQUES}

Bower R. E., Crabo G. B., Pace M. M., Graham E. F., rg73. Effects of dilution and glycerol on the release of glutamic oxaloacetic transaminase (GOT) from boar spermatozoa. J. Anim. Sci., 36, 319-324.

Dacheux J. L., Paguignon M., 1974. Résultats non publiés.

EINARSSON S., SWEnSSON J., ViRING S., 1973. A field trial on the fertility of deep-frozen boar spermatozoa, Nord. Vet. Med., 25, 372-376.

Graham E. F., Crabo B. G., I972. Some factors influencing the freezing of boar spermatozoa, VIIth Int. Congr. Anim. Reprod., Mïnchen, vol II, I627-I632.

Johnson L. A., Gerrits R. J., Young E. I., I969. Quantitative analysis of porcine spermatozoa and seminal plasma phospholipids as affected by frequency of ejaculation. J. Reprod. Fert., 19, 95-102.

du Mesnil du Buisson F., Signoret J. P., i97o. Reproductive physiology and artificial insemination in pigs. Vet. Rec., $8^{\mathrm{m}}, 562-568$.

Paquignon M., du Mesnil. du Buisson F., i973. Fertilité et prolificité de truies inséminées avec du sperme congelé. Journées de la Recherche Porcine en France, 49-57.

Paquignon M., Mergounis D., Courot M., du Mesnil du Buisson F., I974. Technologie de la congélation de la semence de verrat : Étude in vitro. Journées de la Recherche Porcine en France, $7 \mathrm{I}-76$.

Paquignon M., Courot M., i975. Fertilité et prolificité de truies inséminées avec du sperme congelé. Ann. Zootech. (à paraître).

Polge C., Salamon S., Wilmut I., I970. Fertilizing capacity of frozen boar semen following surgical insemination. Vet. Rec., 87, 424-428.

Pursel V. G., Johnson L. A., Schulman L. L., I973. Effect of dilution, seminal plasma and incubation period on cold shock susceptibility of boar spermatozoa. J. Anim. Sci., 27, 528-531.

Pursel V. G., Johnson L. A., I974. Frozen boar spermatozoa : Fertility with concentrated semen and a new thawing procedure. J, Anim. Sci., 39, 222 (Äbstr.).

Rohloff D., Allmeling G., 1972. Zur Tiefgefrierung von Ebersamen. Berliner und Münchener Tierärztliche Wochenschrift., 17, 330-332.

Salamon S., 1973. Deep freezing of boar semen. III. Effects of centrifugation, diluent and dilution rate, pellet volume, and method of thawing on survival of spermatozoa. Aust. J. Biol. Sci., 26, 239-247.

Salamon S., Wilmut I., Polge C., I973. Deep freezing of boar semen. I. Effects of diluent composition, protective agents, and method of thawing on survival of spermatozoa. Aust. J. biol. Sci., 26, 219-230.

Visser D., Salamon S., I974. Effect of composition of tris-based diluent on survival of boar spermatozoa following deep freezing. Aust. J. biol. Sci., 26, 485-497.

Swierstra E. E., r973. Communication personnelle.

Wilmut I., Polge C., 1972. The freezing of boar spermatozoa. VIIth. Int. Congr. Anim. Reprod., Müchen, vol. II, I6II-I6I5. 